
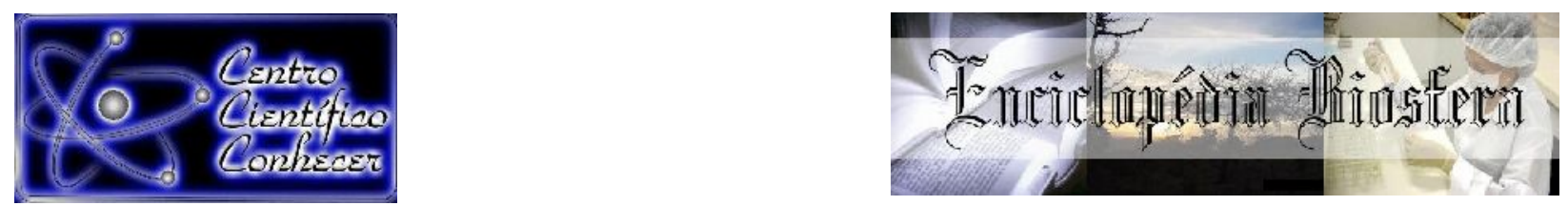

\title{
ANÁLISE ANALÍTICA E NUMÉRICA DE DESLOCAMENTOS DE VIGAS EM CONCRETO ARMADO
}

Fernando Vasconcelos Borba1, Jorcelan Pereira da Rocha²

1 Engenheiro civil. Mestrando em Engenharia de Estruturas e Construção Civil pela Universidade Federal do Pará - UFPA (fernando.ofc@hotmail.com) - Belém, Pará, Brasil.

2 Engenheiro civil. Pós-graduado em Infraestrutura de Transportes e Rodovias pelo Instituto de graduação e pós-graduação - IPOG - Barreirinhas, Maranhão, Brasil.

Recebido em: 15/05/2020 - Aprovado em: 15/06/2020 - Publicado em: 30/06/2020 DOI: 10.18677/EnciBio_2020B49

\begin{abstract}
A grande parte de estruturas usuais de Engenharia Civil tem como utilização o uso de elementos como: lajes, vigas, pilares e fundações. As vigas (elemento estudado neste trabalho) é um elemento estrutural geralmente usado no sistema laje-viga-pilar para transferir os esforços solicitantes verticais oriundos das lajes para os pilares. Portanto, este trabalho tem como principal objetivo apresentar uma análise (comparativa de deslocamentos verticais) analítica e numérica de vigas em concreto armado. Para o modelo analítico, foi empregado o método teórico chamado princípio dos trabalhos virtuais, e para o modelo numérico foi utilizado o software que tem como base o método dos elementos finitos (Ftool). Assim, realizou-se um estudo de caso utilizando o software de dimensionamento de estruturas denominado de Eberick, obtendo os resultados dos deslocamentos das vigas, fazendo assim uma comparação com o método teórico. Conclui-se que, os exemplos analítico e numérico utilizado neste trabalho, deram resultados aproximados, validando assim o modelo teórico descrito nesta análise.
\end{abstract}

PALAVRAS-CHAVE: Deslocamentos. Elementos finitos. Vigas.

\section{ANALYTICAL AND NUMERICAL ANALYSIS OF BEAM DISPLACEMENTS IN REINFORCED CONCRETE}

\begin{abstract}
Most of the usual Civil Engineering structures use elements such as: slabs, beams, pillars and foundations. Beams (element studied in this work) is a structural element generally used in the slab-to-pillar system to transfer the vertical solicitation efforts coming from the slabs to the columns. Therefore, the main objective of this work is to present an analytical and numerical analysis (comparative of vertical displacements) of reinforced concrete beams. For the analytical model, the theoretical method called principle of virtual works was used, and for the numerical model, the software based on the finite element method (Ftool) was used. Thus, a case study using the structure sizing software called Eberick was performed, obtaining the results of the displacements of the beams, thus making a comparison with the theoretical method. It is concluded that the analytical and numerical examples used in this work gave approximate results, thus validating the theoretical model described in this analysis.
\end{abstract}

KEYWORDS: Displacement. Finite elements.Beam. 


\section{INTRODUÇÃO}

As vigas são elementos estruturais lineares quem tem as forças reativas e os momentos fletores resistivos nos apoios, assim, ao se conhecer esses elementos torna-se possível o cálculo dos Momentos Fletores, flechas máximas e outras propriedades (BOTELHO; MARCHETTI, 2015). Timoshenko e Gere (1994) acrescentam que se denomina viga uma barra que suporta cargas transversais em relação ao seu eixo. As cargas em vigas podem ser forças concentradas ou cargas distribuídas ao longo do comprimento da viga. As vigas são usualmente usadas em estruturas de concreto armado e protendido, podendo ser também usado ou fabricado de outro material.

Dessa forma, para Carvalho e Figueiredo Filho (2015) a importância da viga está em transferir os esforços solicitantes verticais oriundos da laje para o pilar. A viga pode ser usada também nas fundações, que tem como principal função de receber as cargas provenientes das paredes e para travamento dos pilares, tornando assim, uma estrutura com estabilidade, funcionalidade e desempenho estrutural.

As vigas devem suportar as cargas solicitantes devendo ser dimensionadas no estado limite último (estado no qual refere-se ao colapso da estrutura) e ao estado limite de serviço (estado no qual refere-se ao desempenho e segurança) devendo ser obedecidos todos os procedimentos de cálculo prescritos nas normas vigentes (ABNT NBR 6118, 2014). Concordando, Porto e Fernandes (2015) citam que as vigas podem ser isostáticas (estruturas nas quais os números de reações são iguais aos números de equações da estática) e hiperestáticas (estruturas nas quais os números de reações são superiores aos números das equações da estática) e hipostáticas (estruturas nas quais os números de reações são menores do que os números das equações da estática) a qual não é usual devido à instabilidade estrutural.

Para colocar em prática esse estudo, é necessário destacar o princípio do trabalho virtual (PTV) que é de grande importância na mecânica das estruturas, proporcionando soluções simples e precisas no cálculo de deslocamentos em estruturas (DIMITROVOVÁ, 2017). Pois de acordo com Assan (1996), o princípio dos trabalhos virtuais pode ser aplicado também às estruturas ou corpos deformáveis, devendo-se levar em consideração não apenas o trabalho virtual das forças externas, mas, também, o associado às forças internas. Durante a deformação virtual, cada elemento (entenda-se elemento diferencial) da estrutura se deforma e os esforços (internos e externos) agindo diretamente sobre ele realizam o trabalho virtual, denominado $d W$ (ASSAN, 1996). Assan (1996) complementa que esse trabalho pode ser supostamente formado por duas parcelas:

$\checkmark$ O trabalho virtual devido aos movimentos de corpo rígido, denominado $d W_{r}$, correspondente ao trabalho virtual realizado durante o movimento de corpo rígido do elemento que assume uma posição intermediária entre uma determinada posição informada;

$\checkmark$ O trabalho virtual referente a deform ação do elemento, designado por $d W_{d}$ Estando o elemento em equilíbrio, o trabalho $d W$, é nulo. Assim, tem-se que:

$$
d W=d W_{d}
$$


A igualdade significa que, durante o deslocamento virtual, o trabalho virtual total realizado pelas forças é igual ao trabalho virtual realizado pelas mesmas forças durante a deformação virtual do elemento. Essa igualdade estendida a toda a estrutura fica:

$$
\int d W=\int d W_{d}
$$

(Equação 2)

A primeira integral da igualdade corresponde apenas ao trabalho virtual das cargas externas, sendo representado por $W_{\text {ext. }}$.

A segunda integral representa o trabalho virtual associado à deformação de todos os elementos da estrutura considerando a ação de todas as forças externas e internas (tensões resultantes). Porém, durante a deformação da estrutura, apenas as forças internas realizam trabalho, correspondendo, então essa integral, ao trabalho virtual interno $W_{\text {int. }}$.

Pode-se dizer, assim, que: o trabalho virtual realizado pelas forças externas, quando se dá a uma estrutura deformável em equilíbrio um deslocamento virtual, é igual ao trabalho realizado pelas forças internas; sendo essa igualdade representada por:

$$
W_{\text {ext }}=W_{\text {int }}
$$

(Equação 3)

O trabalho virtual interno associado a um elemento diferencial é dado por:

$$
d W_{i}=N d u+V d v+M d \theta
$$

Considerando a equação 4 estendida a toda a estrutura e tendo em vista a validade da equação 3 pode-se escrever que:

$$
W_{\text {ext }}=\int N d u+\int V d v+\int M d \theta
$$

(Equação 5)

Além disso, para Assan (1996) o princípio dos trabalhos virtuais aplica-se a estruturas independentemente do material se comportar linearmente ou não, elasticamente ou inelasticamente. Uma aplicação deste método na determinação de deslocamentos em estruturas é o chamado método da carga unitária. Na aplicação desse método são considerados dois sistemas de carregamento. O primeiro sistema consiste na estrutura submetida a ações externas responsáveis pelo deslocamento que se quer calcular.

O segundo sistema de carregamento corresponde à atuação de uma carga unitária agindo na estrutura. Essa carga unitária é fictícia, porém deve corresponder ao deslocamento que se quer calcular (se o deslocamento desejado é uma rotação, a carga fictícia deve ser um momento unitário aplicado no ponto onde se quer a rotação). Seja, pois, $\Delta_{0}$ deslocamento procurado, o trabalho externo produzido pela carga unitária, com o deslocamento desejado $\Delta$, causado por cargas reais vale:

$$
W_{e x t}=1 \cdot \Delta
$$

$\mathrm{O}$ trabalho virtual interno é realizado pelos esforços internos $(\mathrm{N}, \mathrm{V}, \mathrm{M})$, durante a deformação virtual da estrutura, produzida pela carga unitária. Igualando o trabalho virtual externo com 0 interno tem-se determinado o deslocamento procurado:

$$
\Delta=\int N d u+\int V d v+\int M d \theta
$$

Para materiais que seguem a lei de Hooke, as deformações envolvidas na equação 7 são: 


$$
d u=\frac{N_{r} d x}{E A} \quad ; \quad d v=\frac{c V_{r} d x}{G A} \quad ; \quad d \theta=\frac{M_{r} d x}{E I}
$$

Sendo $\mathrm{N}, \mathrm{V}$ e $M_{r}$ as tensões resultantes produzidas pelas cargas reais. Substituindo os valores dados pela equação 2.8 na equação 2.7 tem-se:

$$
\Delta=\int \frac{N_{1} N_{r}}{E A} d x+\int \frac{c V_{1} V_{r}}{G A} d x+\int \frac{M_{1} M_{r}}{E I} d x
$$

De acordo com a norma ABNT (NBR 6118, 2014) o limite de deslocamento é obedecido pela seguinte expressão:

$$
\frac{l}{250}
$$

onde

$l$ : é o vão da viga em centímetros

Basta, então, conhecer os diagramas de esforços produzidos pela carga unitária e pelas cargas reais e combiná-los. O deslocamento $\Delta$ tanto pode ser rotação como translação. Dessa forma, de acordo com as equações supracitadas são possíveis análises comparativas entre duas ou mais estruturas, permitindo uma ampla vantagem aos engenheiros calculistas presentes na atualidade.

\section{Especificações da análise realizada}

\section{MATERIAL E MÉTODOS}

O estudo foi feito em uma estrutura em concreto armado, tendo sapatas e vigas baldrames como fundação. Tem-se para o pavimento superior quatro vigas no total, no qual será calculado o deslocamento máximo de cada uma, para comparar os resultados obtidos no modelo de cálculo via método dos elementos finitos (Ftool) e no software de dimensionamento estrutural (Eberick). Para melhor entendimento, tais programas são definidos das seguintes formas:

Eberick é um programa de cálculo estrutural que facilita a visualização da geometria de uma estrutura através da criação em pórticos 3D. Ou seja, apresenta um ambiente de CAD próprio, com soluções específicas para modelagem de pilares, vigas, lajes, escadas, fundações, reservatórios, muros e outros elementos de diversos materiais (ALTOQI, 2020).

Ftool é um software que serve para a verificação de estruturas planas. Deixando de ser um simples programa utilizado em sala de aula, e tornou-se uma ferramenta indispensável em análise estrutural, porquanto, tem a finalidade de criar um protótipo simples e eficiente de estruturas. Dessa forma, esse programa possibilita uma verificação do modelo estrutural, tais como: diagramas de forças internas e configuração deformada das estruturas, assim, como linhas de influência e envoltórias decorrentes do trem de carga (FTOOL, 2016). Entretanto, antes de qualquer conclusão da pesquisa final, são apresentados a seguir modelos analíticos e numéricos com objetivo de esclarecer todo procedimento da análise.

\section{Modelo analítico 1}

O objetivo do exemplo a seguir, figura 1, é calcular o deslocamento vertical no meio da viga. Será calculado passo a passo, para mostrar o modelo de cálculo na prática. Será adotado uma viga engastada de 12 metros de comprimento com um 
carregamento distribuído à quatro metros do engaste, e a quatro metros da extremidade à direita. Dados da viga: $b_{w}=40 \mathrm{~cm} ; h=100 \mathrm{~cm} ; E=25 G P a$.

FIGURA 1- Carregamento real produzido

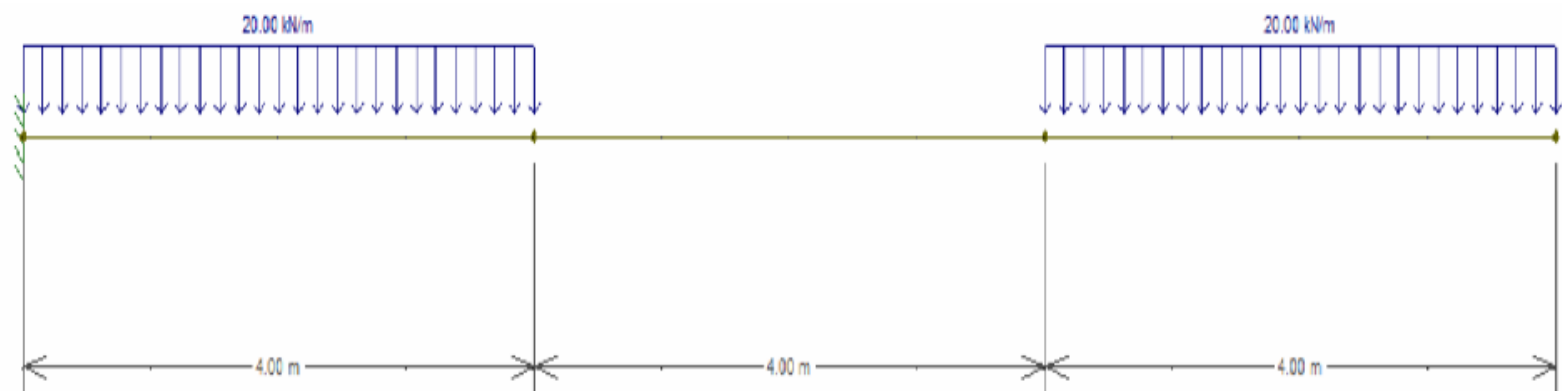

Fonte: Captura de tela do software Ftool (2019)

São realizados três cortes transversais para poder obter os esforços da viga. Partindo-se do princípio para o cálculo das reações de apoio. Na figura 2 tem-se o sistema real da estrutura.

FIGURA 2 - Sistema real com cortes transversais

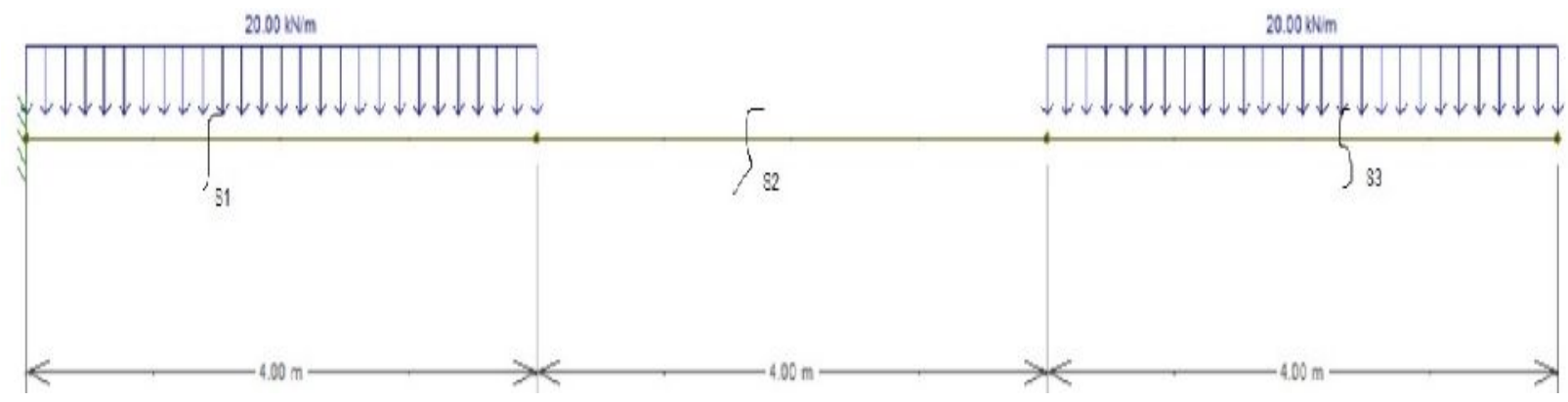

Fonte: Captura de tela do software Ftool (2019)

Tem-se as reações de apoio:

$$
\begin{aligned}
& \sum M_{A}=0 \\
& -80 \cdot 2-80 \cdot 10+M=0 \\
& M=960 \mathrm{kNm} \\
& \sum F y=0 \\
& -80-80+A y=0 \\
& A y=160 \mathrm{kN}
\end{aligned}
$$


Assim, obtém-se as equações dos momentos fletores do sistema real da estrutura. Para o corte transversal s1 pela esquerda tem-se a seguinte equação de momento fletor:

$\{0 \leq x \leq 4 m\}$

$\sum M_{s 1}=0$

$-160 \cdot x+20 \cdot x \cdot \frac{x}{2}+960+M_{s 1}=0$

$M_{s 1}=160 x-10 x^{2}-960 k N m$

Para o corte transversal s2 tem-se o seguinte resultado:

$\{4 \leq x \leq 6 m\}$

$\sum M_{s 2}=0$

$-160 \cdot x+80 k N \cdot(x-2 m)+960+M_{s 2}=0$

$M_{s 2}=80 x-800 k N m$

Realizando um corte transversal s2 pela direita para se obter uma equação para ser compatibilizado as equações do sistema real e virtual. Portanto, tem-se:

$\{4 \leq x \leq 6 m\}$

$\sum M_{s 2 d i r}=0$

$-M-80 \cdot(x-2)=0$

$M=-80 x+160 \mathrm{kNm}$

Por fim, Tem-se o corte s3:

$\{0 \leq x \leq 4 m\}$

$\sum M_{s 3}=0$

$-M-20 x \cdot \frac{x}{2}=0$

$M=-10 x^{2}$

No sistema virtual: Retiram-se todas as cargas existentes, deixando apenas os dados geométricos. Para calcular o deslocamento no meio da viga, necessita-se colocar uma carga unitária no ponto onde se deseja calcular o deslocamento. Portanto, tem-se a seguinte situação:

FIGURA 3 - Carregamento virtual

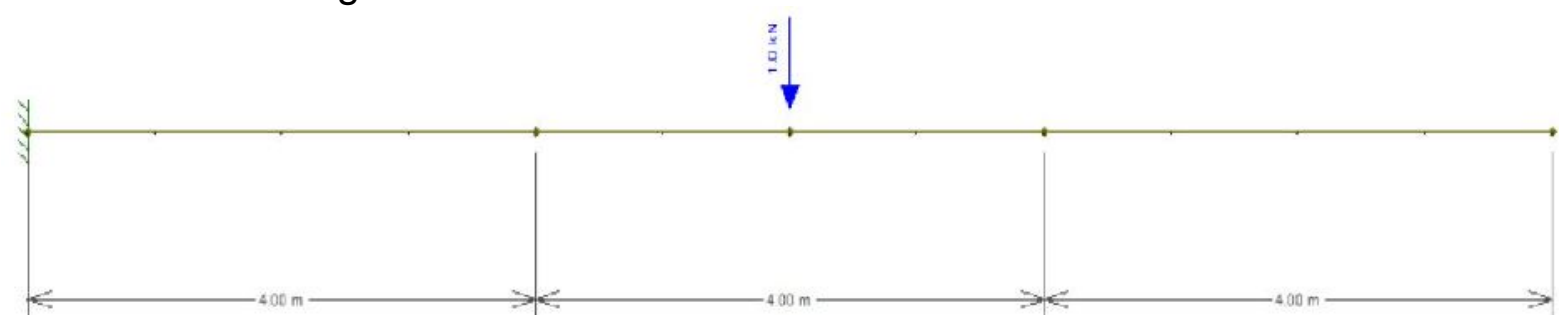

Fonte: Captura de tela do software Ftool (2019)

Serão adotados os mesmos cortes utilizados no sistema real, para se utilizar o método com grande precisão e assertividade. 
FIGURA 4 - Sistema real com cortes transversais

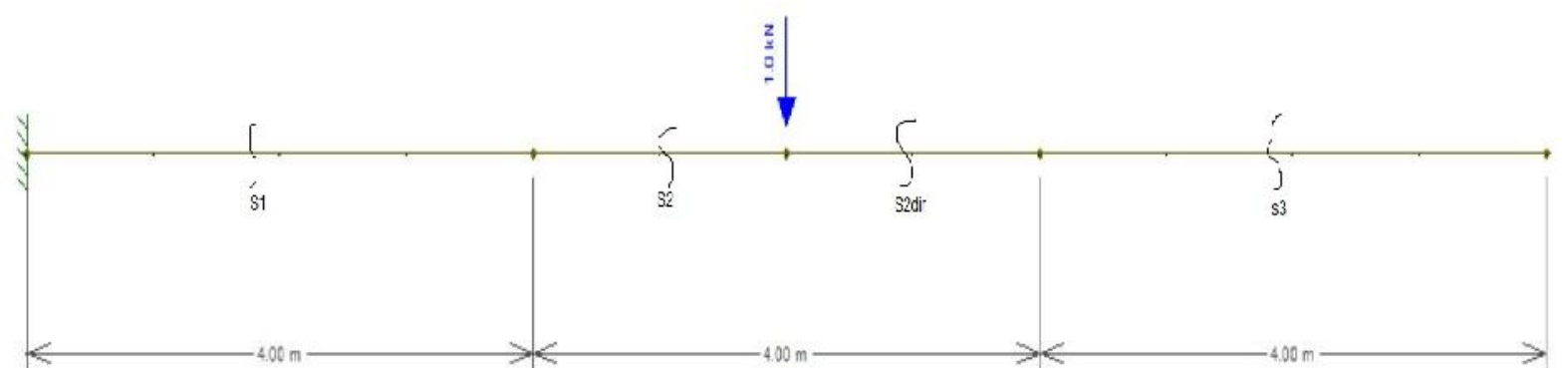

Fonte: Captura de tela do software Ftool (2019)

Nas reações de apoio, obtém-se:

$\sum M_{A}=0$

$\sum F y=0$

$-1 \cdot 6+M=0$

$A y-1=0$

$M=6 \mathrm{kNm}$

$A y=1 k N$

Nas equações dos momentos fletores do sistema virtual da estrutura, para o corte transversal s1 pela esquerda, tem-se a seguinte equação de momento fletor:

$\{0 \leq x \leq 4 m\}$

$\sum M_{s 1}=0$

$6-1 \cdot x+M_{s 1}=0$

$M_{s 1}=x-6 \mathrm{kNm}$

Para o corte transversal s2 pela esquerda, tem-se a seguinte equação de momento fletor:

$\{4 m \leq x \leq 6 m\}$

$\sum M_{s 2}=0$

$6-1 \cdot x+M_{s 2}=0$

$M_{s 2}=x-6 \mathrm{kNm}$

Para o corte transversal s2 pela direita, tem-se a seguinte equação de momento fletor:

$\{4 m \leq x \leq 6 m\}$

$\sum M_{s 2}=0$

$M_{\text {s2dir }}=0$

Para o corte transversal s3 pela direita, tem-se a seguinte equação de momento fletor:

$\{0 \leq x \leq 4 m\}$

$\sum M_{s 3}=0$

$M_{s 3}=0$

Para os cálculos do deslocamento será utilizado a fórmula de princípio dos trabalhos virtuais, considerando apenas deslocamento por flexão, devido em vigas o esforço preponderante ser o de flexão. Nos exemplos posteriores será apresentado um exemplo, no qual será considerado o esforço cortante no cálculo do deslocamento. 
Onde:

(Equação 11)

$$
\Delta=\int \frac{M_{1} M_{r}}{E I} d x
$$

$M_{r}$ é o momento real, ${ }^{M_{1}}$ é o momento virtual, E é o módulo de elasticidade do concreto, I é o momento de inércia, o qual é calculado pela fórmula a seguir:

$$
I=\frac{b \cdot h^{3}}{12}
$$

(Equação 12)

Portanto, o deslocamento no meio da viga será:

$$
\begin{aligned}
& \Delta_{\text {Meio }}=\frac{1}{E I}+\int_{0}^{4}\left(160 x-10 x^{2}-960\right) \cdot(\mathrm{x}-6) \mathrm{dx}+\int_{4}^{6}(80 x-800) \cdot(x-6) d x+\int_{0}^{4}\left(-10 \mathrm{x}^{2}\right) \cdot(0) d x+\int_{4}^{6}(-80 x+160) \cdot(0) d x \\
& \Delta_{\text {Meio }}=\frac{12586,663}{E I}=\frac{12586,663}{25 \cdot 10^{6} \cdot 0,03333}=0.0151 \mathrm{~m}=-1.51 \mathrm{~cm}
\end{aligned}
$$

Conclui-se que, de acordo com os carregamentos e geometria da viga, tem-se um deslocamento de 1.51 centímetros no meio desta.

\section{Modelo analítico 2}

Será apresentado um exemplo de uma viga biapoiada com carregamento distribuído, com vão de oito metros, levando em consideração os esforços de momento fletor e esforço cortante. Será calculado o deslocamento no meio da viga adotando as formulações matemáticas descritas anteriormente:

$$
\text { Dados da viga: } b_{w}=40 \mathrm{~cm}, h=80 \mathrm{~cm}, E=24.855 \mathrm{GPa} \text {. }
$$

FIGURA 5- Carregamento produzido

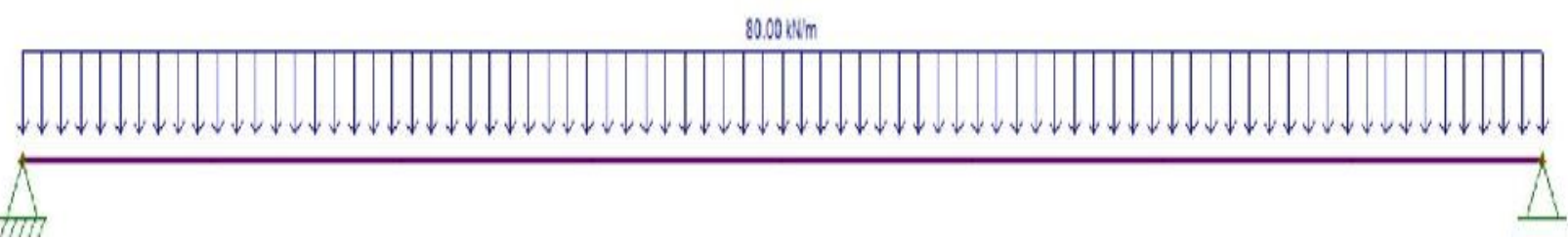

Fonte: Captura de tela do software Ftool (2019)

Para esforço cortante, tem-se o resultado final do deslocamento:

$$
\int \frac{c V_{1} V_{r}}{G A} d x=0.023 \mathrm{~cm}
$$

Para o momento fletor, tem-se o resultado final do deslocamento:

$$
\int \frac{M_{1} M_{r}}{E I} d x=1.006 \mathrm{~cm}
$$

Somando o deslocamento causado pelo esforço cortante, com o deslocamento causado pelo momento fletor, tem-se o deslocamento total no meio da viga:

$$
\Delta_{\text {Total }}=1.03 \mathrm{~cm}
$$




\section{Modelo numérico 1}

Será apresentado agora a mesma viga do modelo analítico 1 modelando a mesma no software Ftool, com o objetivo de comparar o resultado analítico com o numérico (computacional).

FIGURA 6 - Carregamento real produzido

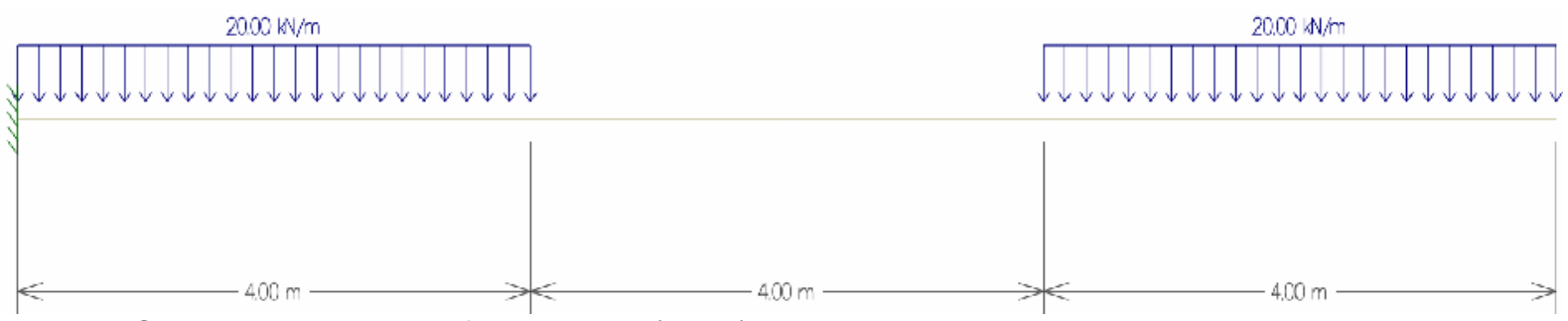

Fonte: Captura de tela do software Ftool (2019)

O resultado apresentado pelo software é de 1,51 centímetro, o mesmo apresentado pelo modelo teórico do princípio dos trabalhos virtuais. Portanto, podese concluir, que os resultados obtidos no modelo analítico e numérico são iguais, validando assim ambos os modelos para a utilização na engenharia estrutural.

FIGURA 7 - Deslocamento no meio da viga

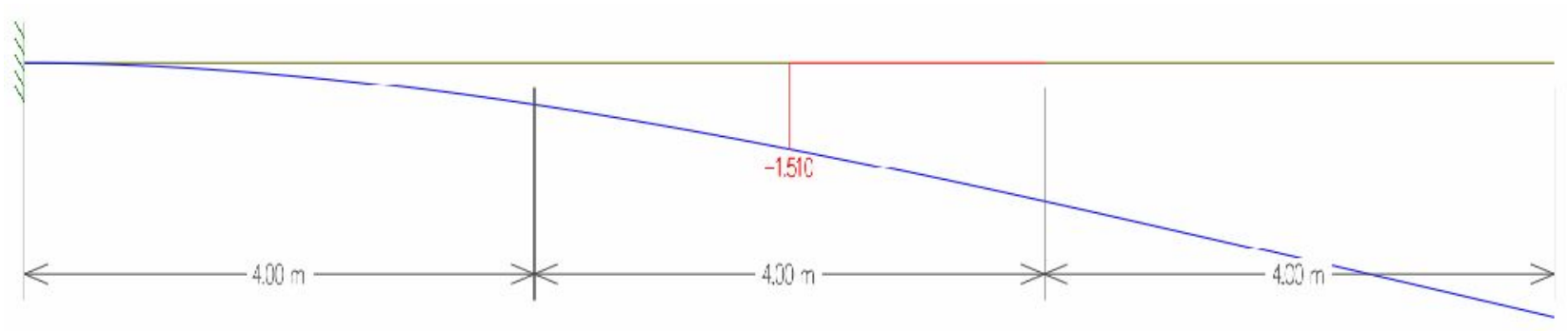

Fonte: Captura de tela do software Ftool (2019)

\section{Modelo numérico 2}

Será apresentada a mesma viga do modelo analítico 2 , modelando a mesma no software Ftool, com o objetivo de comparar o resultado analítico com o numérico (computacional).

FIGURA 8 - Deslocamento no meio da viga

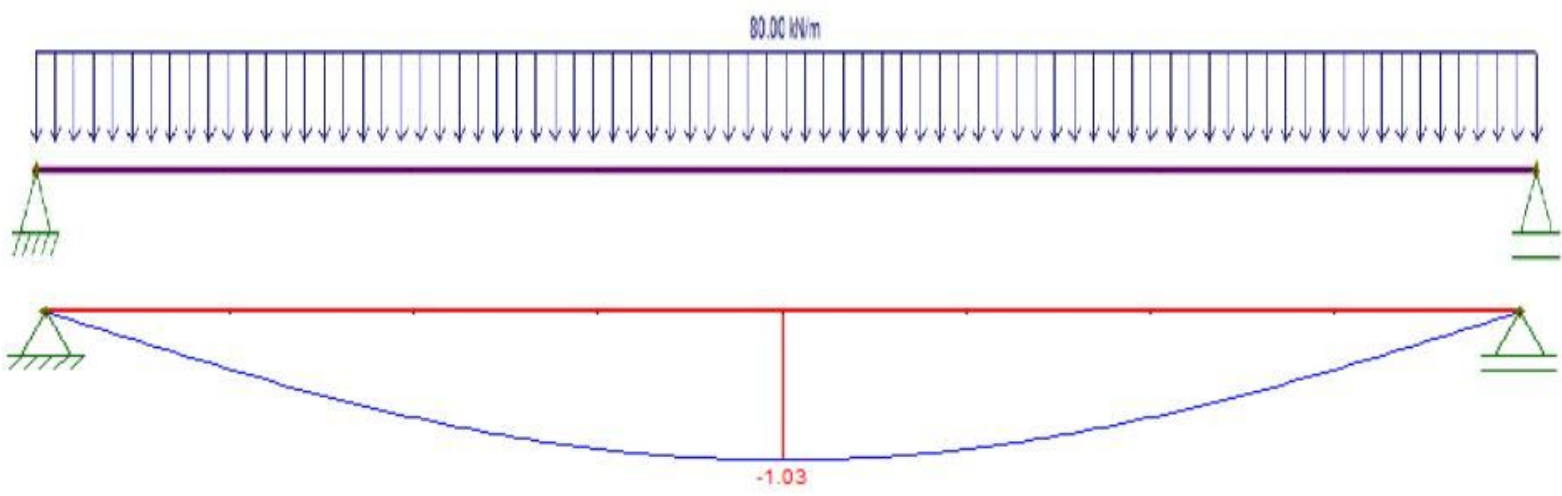

Fonte: Captura de tela do software Ftool (2019) 
Percebe-se, que os resultados obtidos no cálculo manual e pelo software são iguais, validando novamente ambos os modelos de cálculos. Pode-se perceber que o resultado total do deslocamento da viga equivalente a 1.03 centímetros, é o somatório dos deslocamentos causado pelo momento fletor e esforço cortante. Portanto, os modelos de cálculo (analítico e numérico) apresentados neste trabalho, são de extrema importância, pois, ambos os modelos apresentam valores iguais.

\section{RESULTADOS E DISCUSSÃO}

Para que se obtenha um resultado preciso e em curto período de tempo, é preciso utilizar softwares, por exemplo, Eberick que permite criar um memorial de cálculo da edificação, através dos critérios adotados pelo projetista, dentro das normas utilizadas na análise. Gerando relatórios que permitem a análise da verificação global, dos esforços e do dimensionamento dos elementos e cargas nas fundações (ALTOQI, 2020).

Assim, na obtenção de um diagnóstico mais amplo e resultados mais precisos, verificaram-se quatro vigas (modelagem no programa Eberick), obtendo-se resultados no modelo analítico (cálculo manual) e numérico (Ftool). As cargas nas vigas V1 e V3 equivale à $600 \mathrm{~kg} / \mathrm{m}$, e as cargas nas vigas V2 e V4 iguais à 1817,27 $\mathrm{kg} / \mathrm{m}$. As vigas têm seção transversal de $(15 \times 30) \mathrm{cm}$, Fck=30MPa e $E=26320 \mathrm{MPa}$. $\mathrm{Na}$ figura 9 é possível fazer uma verificação detalhada da estrutura de concreto armado em estudo.

FIGURA 9 - Estrutura em concreto armado

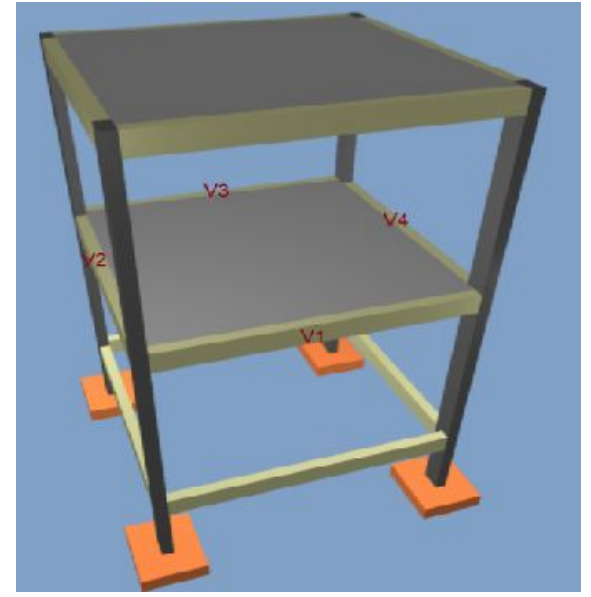

Fonte: Captura de tela do software Eberick (2019)

Na obtenção desses resultados, Dimitrovová (2017) cita que é necessário entender que o deslocamento de uma partícula ou de um ponto que pertence a um corpo rígido, representa a alteração de posição que muitas vezes passa despercebida aos usuários das edificações, entretanto, para análise estrutural é fundamental conhecer.

De acordo com Assan (1996), entre outros métodos difundidos para obtenção de deslocamentos em vigas, com o PTV se obtém um deslocamento infinitesimal que se impõe a um ponto ou a um corpo rígido de modo a não alterar a configuração estática ou geométrica do corpo e das forças que neste atuam, preservando as condições de equilíbrio a que essas forças estão sujeitas. Um deslocamento virtual consiste em uma translação em qualquer direção, uma rotação em torno de qualquer 
eixo ou ambos. Com a condição de o corpo rígido estar em equilíbrio, o trabalho virtual realizado pelas forças é nulo.

Dessa maneira, se evidencia que os dados "finais" são alcançados através de diferentes formas (manuais e computacionais), entretanto, com o desenvolvimento de programas é possível uma verificação mais rápida e dinâmica (através de modelagem), como evidenciado nos resultados obtidos neste trabalho. Na figura 10 é mostrado o pórtico unifilar, onde é mostrada com clareza na cor avermelhada à simulação dos deslocamentos das vigas provocados pelos carregamentos verticais.

FIGURA 10 - Pórtico unifilar

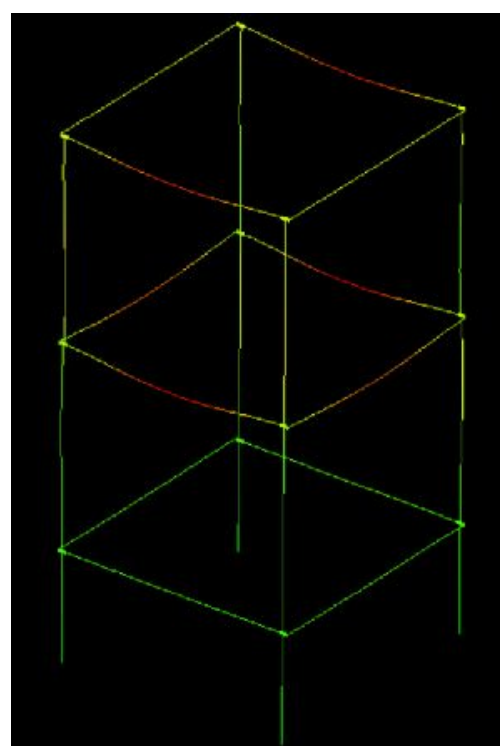

Fonte: Captura de tela do software Eberick (2019)

Paralelamente, Botelho e Marchetti (2015) mencionam que nos estudos sobre o deslocamento de vigas biapoiadas, a parte inferior do concreto fissura (sem causar a degradação da estrutura), porquanto, quem está trabalhando à tração é o aço. Destaca-se também, que todo o processo da análise de vigas que sofrem pequenos deslocamentos, está ligado diretamente às condições de solicitação e cálculo de viga.

\section{Resultados obtidos pelo software Eberick}

Nas figuras 11 e 12 é possível observar a aplicação das cargas nos elementos estruturais. 
FIGURA 11- Disposição das vigas e devidas cargas

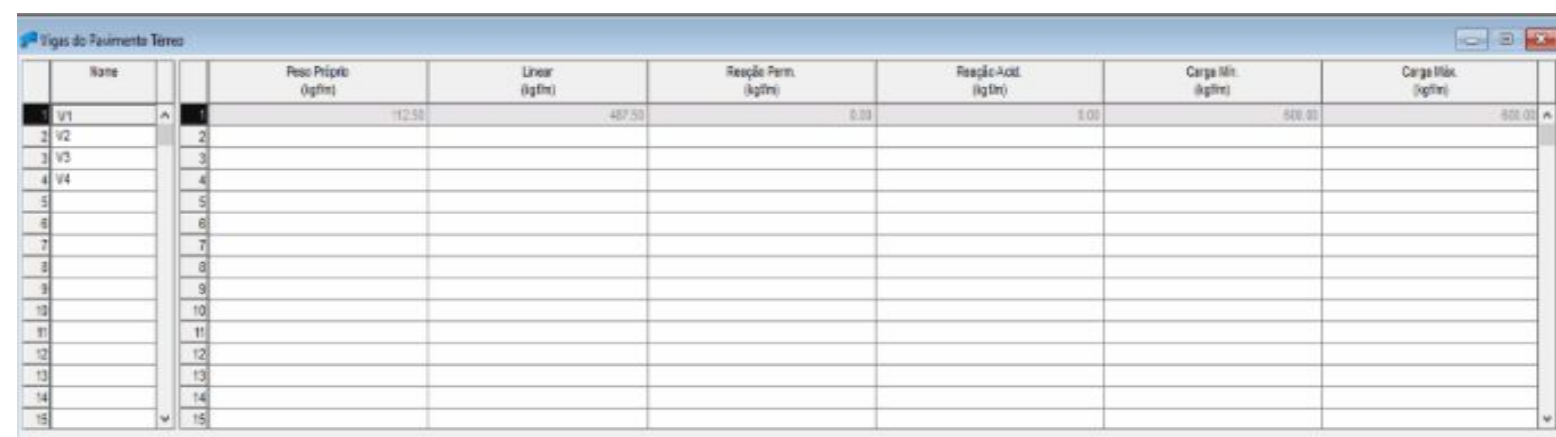

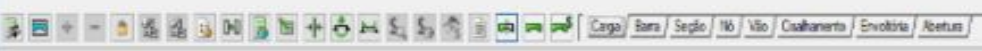

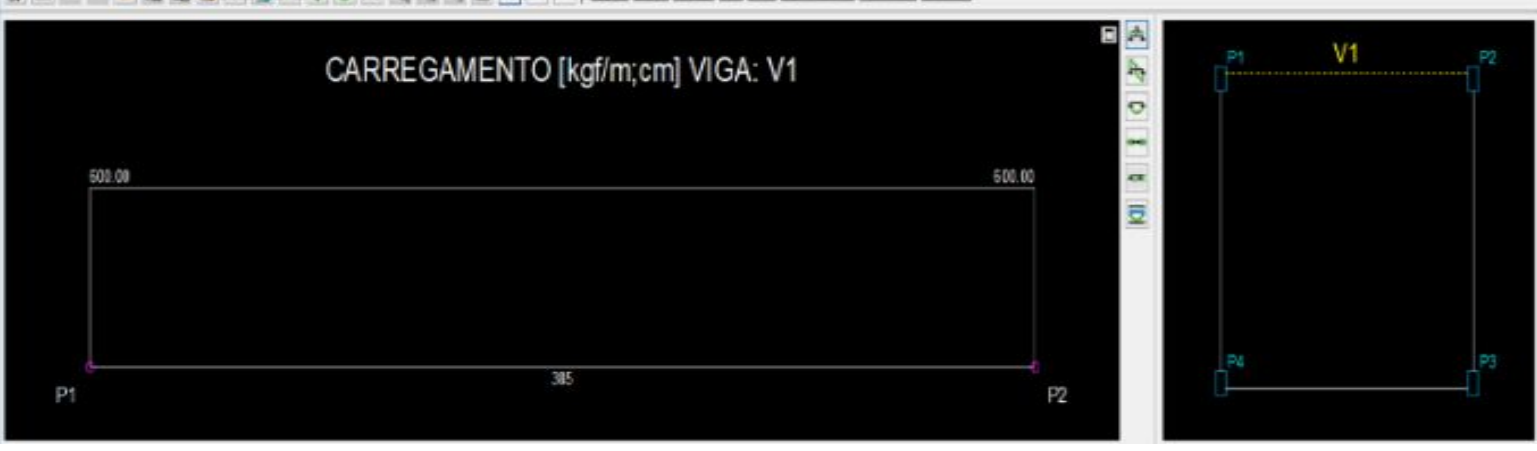

Fonte: Captura de tela do software Eberick (2019)

FIGURA 12 - Disposição das vigas e devidas cargas
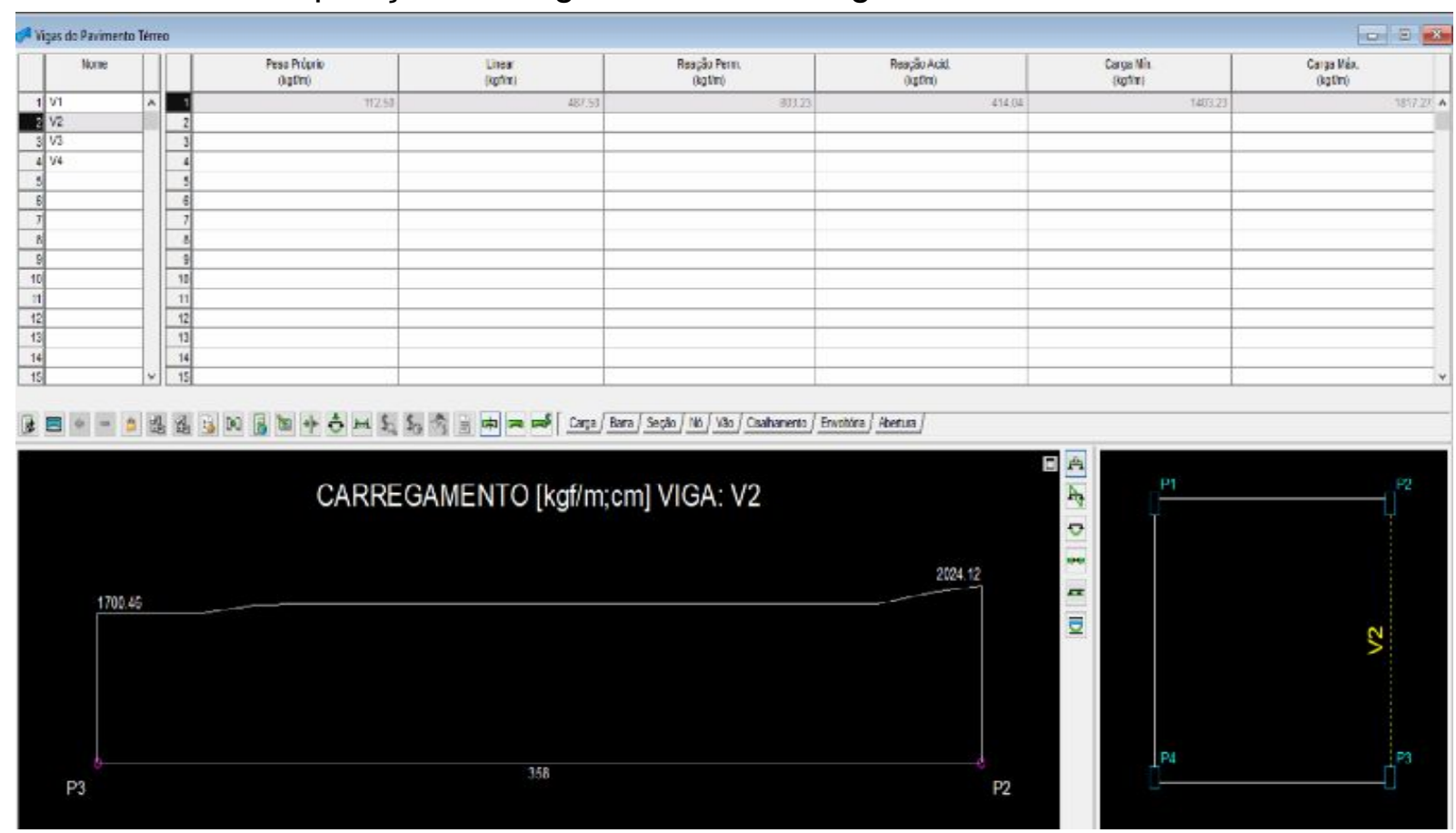

Fonte: Captura de tela do software Eberick (2019)

$\mathrm{Na}$ figura 13, é notório os resultados dos deslocamentos totais das vigas obtidas pelo software Eberick. 
FIGURA 13 - Deslocamento das vigas

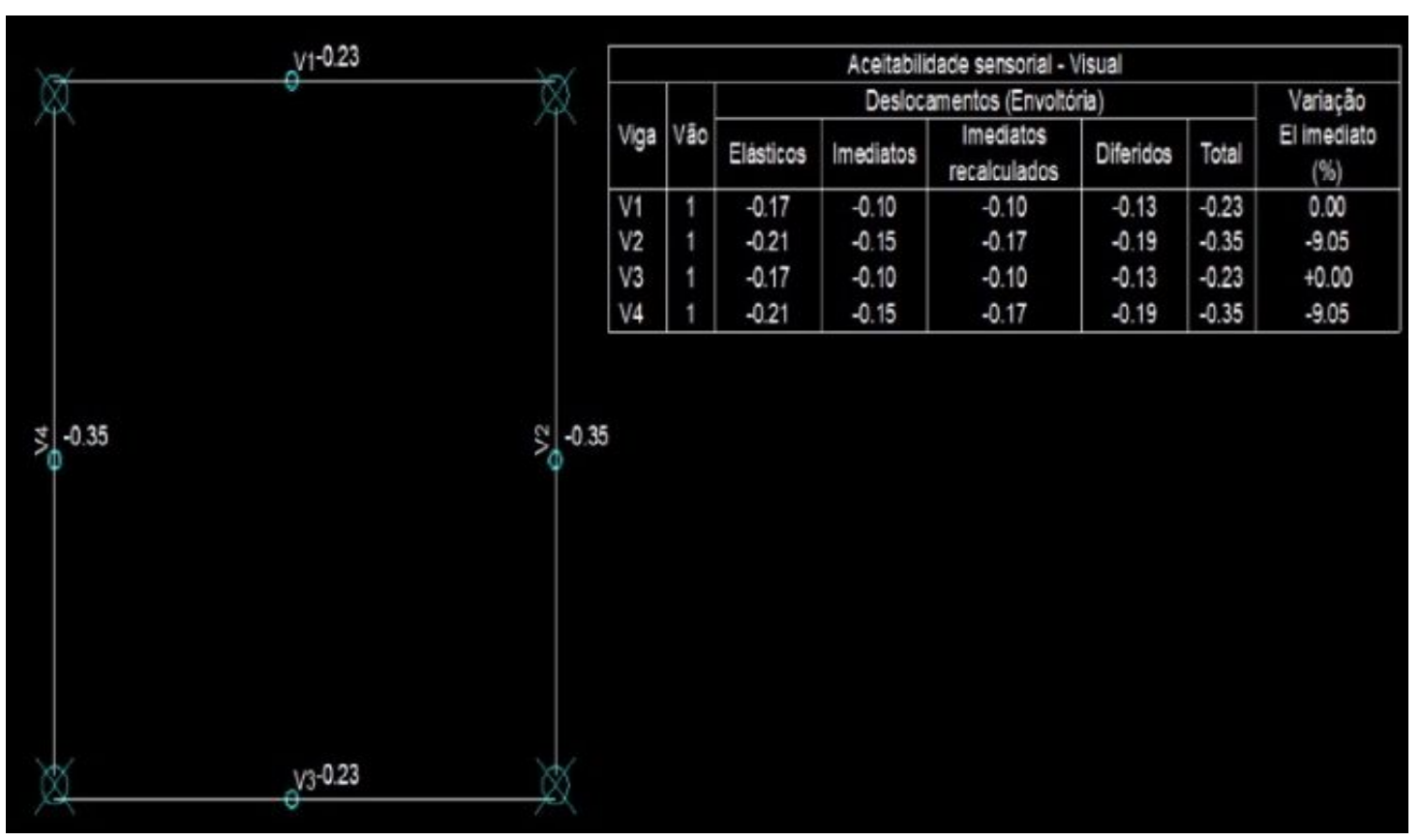

Fonte: Captura de tela do software Eberick (2019)

Resultados dos deslocamentos das vigas obtidos pelo Eberick

TABELA 1 - Deslocamento obtido pelo software Eberick

\begin{tabular}{|c|c|c|c|}
\hline VIGA & VÃO $(\mathbf{m})$ & CARGA $\mathbf{( k g f} / \mathbf{m})$ & FLECHA TOTAL $(\mathbf{c m})$ \\
\hline V1 & 4 & 600 & 0,23 \\
\hline V2 & 4 & 1817,27 & 0,35 \\
\hline V3 & 4 & 600 & 0,23 \\
\hline V4 & 4 & 1817,27 & 0,35 \\
\hline
\end{tabular}

Fonte: Resultado da pesquisa (2019)

Resultados dos deslocamentos das vigas obtidos pelo ftool

FIGURA 14 - Deslocamento das vigas V1 e V2
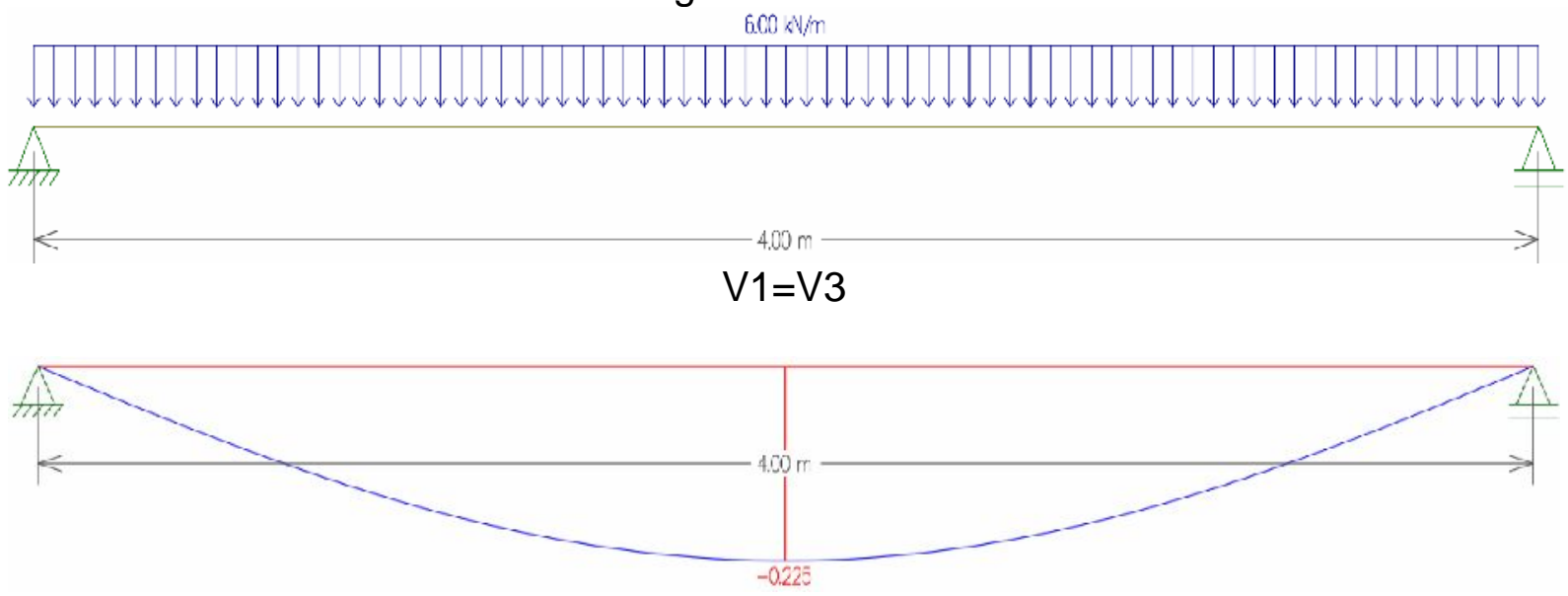

Fonte: Resultado da pesquisa (2019) 
FIGURA 15 - Deslocamento das vigas V2 e V4
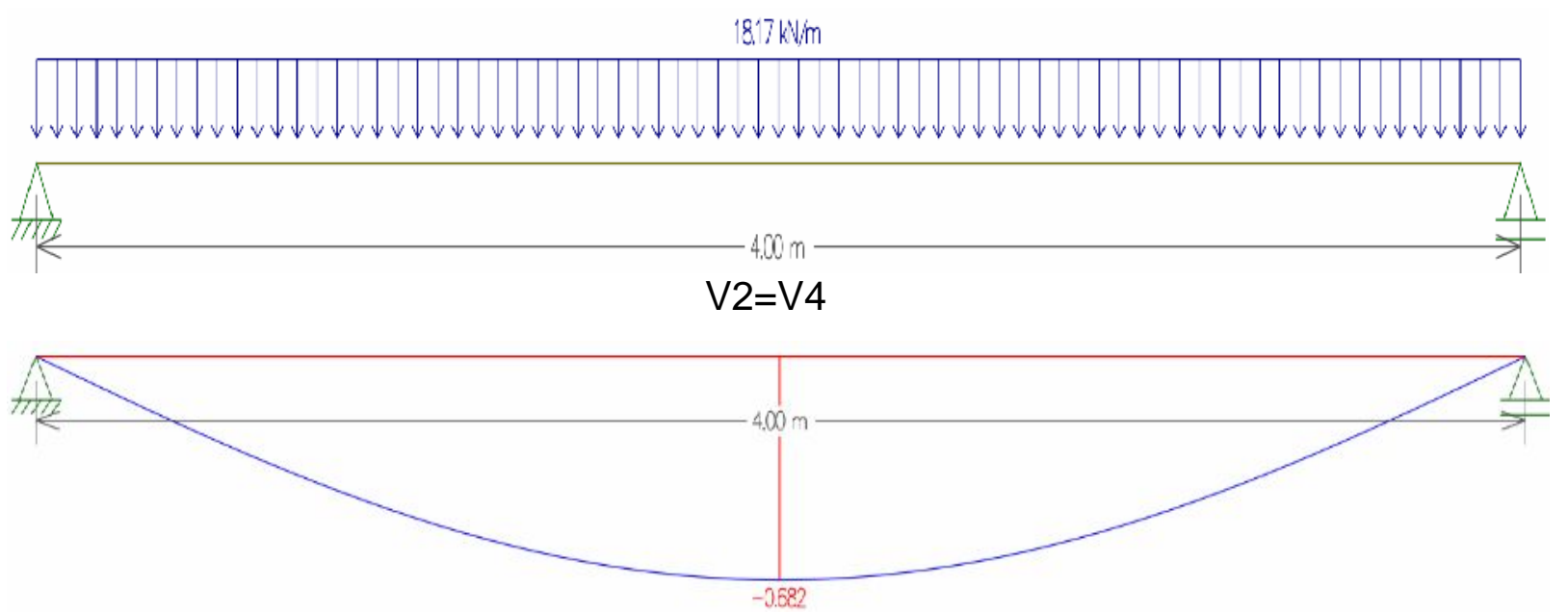

Fonte: Resultado da pesquisa (2019)

TABELA 2 - Deslocamento obtido pelo software ftool

\begin{tabular}{|c|c|c|c|}
\hline VIGA & VÃO $(\mathbf{m})$ & CARGA $(\mathbf{k g f} / \mathbf{m}$ & FLECHA TOTAL $(\mathbf{c m})$ \\
\hline V1 & 4 & 600 & 0,225 \\
\hline V2 & 4 & 1817,27 & 0,682 \\
\hline V3 & 4 & 600 & 0,225 \\
\hline V4 & 4 & 1817,27 & 0,682 \\
\hline
\end{tabular}

Fonte: Resultado da pesquisa (2019)

\section{CONCLUSÃO}

Pode-se concluir que, as comparações em geral foram satisfatórias, validando assim tanto o modelo analítico (cálculo manual), quanto ao modelo numérico (cálculo pelos softwares). No estudo de caso, somente nas vigas V2 e V4, o software de dimensionamento Eberick apresentou um valor de deslocamento equivalente à 0,35 centímetros, um valor diferente obtido no software Ftool que apresentou um valor igual à 0,68 centímetros. Percebeu-se que tal diferença se deu, devido a essas vigas servirem de apoio da laje (vigotas unidirecionais apoiadas nas vigas V2 e V4) suportando assim cerca de $75 \%$ das cargas do pavimento, e apenas cerca de $25 \%$ para as vigas V1 e V3. Por esse motivo, o software de dimensionamento Eberick utilizou várias combinações de cargas, o qual obteve uma mais útil para as devidas vigas, resultando assim em um deslocamento menor ao obtido no software Ftool.

\section{REFERÊNCIAS}

ALTOQI. Solução para seus projetos estruturais. 2020. Disponível em: <https://www.altoqi.com.br/eberick/>. Acesso em: 05/02/2020.

ASSAN, A.E. Método dos Elementos Finitos: Primeiros passos. Editora da Unicamp, Campinas, 1996.

ABNT - Associação Brasileira de Normas Técnicas. NBR 6118: Projeto de estruturas de concreto - procedimento. Rio de Janeiro: ABNT, 2014. 
BOTELHO, M. H. C; MARCHETTI, O. Concreto Armado - Eu te Amo. v. 1. São Paulo. Blucher, 2015.

CARVALHO, R. C.; FIGUEIREDO FILHO, J. R.F. Cálculo e Detalhamento de Estruturas Usuais de Concreto Armado. 4. ed. São Carlos. Editora Edufscar, 2015.

DIMITROVOVÁ, Z. Notas de Aula: O Princípio dos Trabalhos Virtuais. Departamento de Engenharia civil da Universidade Nova de Lisboa, Lisboa, 2017. Disponível $<$ http://www2.dec.fct.unl.pt/seccoes/S_Estruturas/DCR/teoricas/2_1Sebenta.pdf>. Acesso em: 04/02/2020.

FTOOL. Ftool. 2016. Disponível em: <https://www.ftool.com.br/Ftool/site/about>. Acesso em: 05/02/2020.

PORTO, T. B.; FERNANDES, D. S. G. Curso básico de concreto armado: conforme NBR 6118/2014. São Paulo. Editora Oficina de textos, 2015.

TIMOSHENKO, S.P.; GERE, J. E. Mecânica dos Sólidos. Rio de Janeiro. Livros Técnicos e Científicos,1994. 